\title{
Anesthesia management of atrial myxoma resection with multiple cerebral aneurysms: a case report and review of the literature
}

\author{
Ran Zhang ${ }^{1}$, Zhiyu Tang ${ }^{1}$, Qing Qiao ${ }^{1}$, Feroze Mahmood ${ }^{2}$ and Yi Feng ${ }^{1 *}$ (I)
}

\begin{abstract}
Background: Embolic stroke is a common complication of atrial myxoma, whereas multiple cerebral aneurysms associated with atrial myxoma is rare. The pathogenesis of the cerebral vascular disease related to an atrial myxoma is still not well known, and there are no guidelines to guide treatment and anesthesia management in such patients.

Case presentation: In this report, we present a 38-year-old woman with occasional dizziness and headache diagnosed as multiple cerebral fusiform aneurysms, in whom transthoracic echocardiography revealed a mass attached to the interatrial septum in the left atrium. Myxoma resection was performed in fast track cardiac surgery pathway without neurological complications, and no intervention was carried out on the cerebral aneurysms. She was discharged home 6 days after the procedure for followed-up. Furthermore, we reviewed and analyzed the literature in the PubMed and Google Scholar databases in order to conclude the optimal treatment in such cases.

Conclusions: Atrial myxoma-related cerebral aneurysms are always multiple and in a fusiform shape in most occasions. Early resection of myxoma and conservative therapy of aneurysm is an optimal treatment. TEE and $\mathrm{PbtO}_{2}$ monitoring play an essential role in anesthesia management. Fast track cardiac anesthesia is safe and effective to early evaluate neurological function. Long term follow-up for "myxomatous aneurysms" is recommended. And outcome of most patients is excellent.
\end{abstract}

Keywords: Multiple cerebral aneurysms, Atrial myxoma, Anesthesia management

\section{Background}

Atrial myxoma is the most common benign cardiac tumor, which represents about $50 \%$ of all primary cardiac tumors. Approximately $75 \%$ occur in the left atrium [1]. Systemic embolism due to atrial myxoma has been well documented, especially embolic stroke [2]. However, intracranial aneurysms are rarely associated to atrial myxoma [3]. We present the case of a woman with dizziness and headache whose brain computed tomography angiography (CTA) manifested multiple fusiform aneurysms,

\footnotetext{
* Correspondence: doctor_yifeng@sina.com

'Department of Anesthesiology, Peking University People's Hospital, No. 11 Xi Zhi Men Nan Da Jie, Xicheng District, Beijing, China

Full list of author information is available at the end of the article
}

and transthoracic echocardiography revealed a mass in the left atrium.

The pathogenesis of the cerebral vascular disease related to an atrial myxoma is still not well known, and there are no guidelines to guide treatment and anesthesia management in such patients.

\section{Case presentation}

Case report

A 38-year-old woman with no medical history presented 10 days of dizziness and headache without loss of consciousness, dysarthria, weakness, nausea, or vomiting. Neurological examination was normal. The brain CTA manifested two unruptured fusiform aneurysms, which

(c) The Author(s). 2020 Open Access This article is licensed under a Creative Commons Attribution 4.0 International License, which permits use, sharing, adaptation, distribution and reproduction in any medium or format, as long as you give appropriate credit to the original author(s) and the source, provide a link to the Creative Commons licence, and indicate if changes were made. The images or other third party material in this article are included in the article's Creative Commons licence, unless indicated otherwise in a credit line to the material. If material is not included in the article's Creative Commons licence and your intended use is not permitted by statutory regulation or exceeds the permitted use, you will need to obtain permission directly from the copyright holder. To view a copy of this licence, visit http://creativecommons.org/licenses/by/4.0/ The Creative Commons Public Domain Dedication waiver (http://creativecommons.org/publicdomain/zero/1.0/) applies to the data made available in this article, unless otherwise stated in a credit line to the data. 
located in left anterior cerebral artery and left posterior cerebral artery, with the size of $9.7 \mathrm{~mm} \times 6.3 \mathrm{~mm}$ and $10.2 \mathrm{~mm} \times 7 \mathrm{~mm}$, respectively (Fig. 1). Furthermore, transthoracic echocardiography (TTE) revealed a $4.8 \times$ $2.9 \times 2.5 \mathrm{~cm}^{3}$ mass attached to the interatrial septum in the left atrium, which obstructed the mitral orifice without mitral valve regurgitation (Fig. 2).

According to the recommendation of multidisciplinary team (MDT), myxoma was first considered to be excised, a conservative approach was chosen for cerebral aneurysms, and the fast track cardiac surgery pathway should be performed to evaluate neurological function as soon as possible. The baseline vital signs of this patient were measured before induction of general anesthesia, in order to maintain the fluctuation range of heart rate (HR) and mean arterial pressure (MAP) within 10\% throughout the perioperative period. The mass was successfully removed and histological examination confirmed a typical myxoma (Fig. 3). No mitral regurgitation or shunt flow across the atrial septum was revealed by transesophageal echocardiography (TEE) (Fig. 4). Parenchymal brain oxygen $\left(\mathrm{PbtO}_{2}\right)$ monitoring did not change significantly throughout the procedure. The patient was transported to intensive care unit (ICU) receiving infusion of propofol. After that,

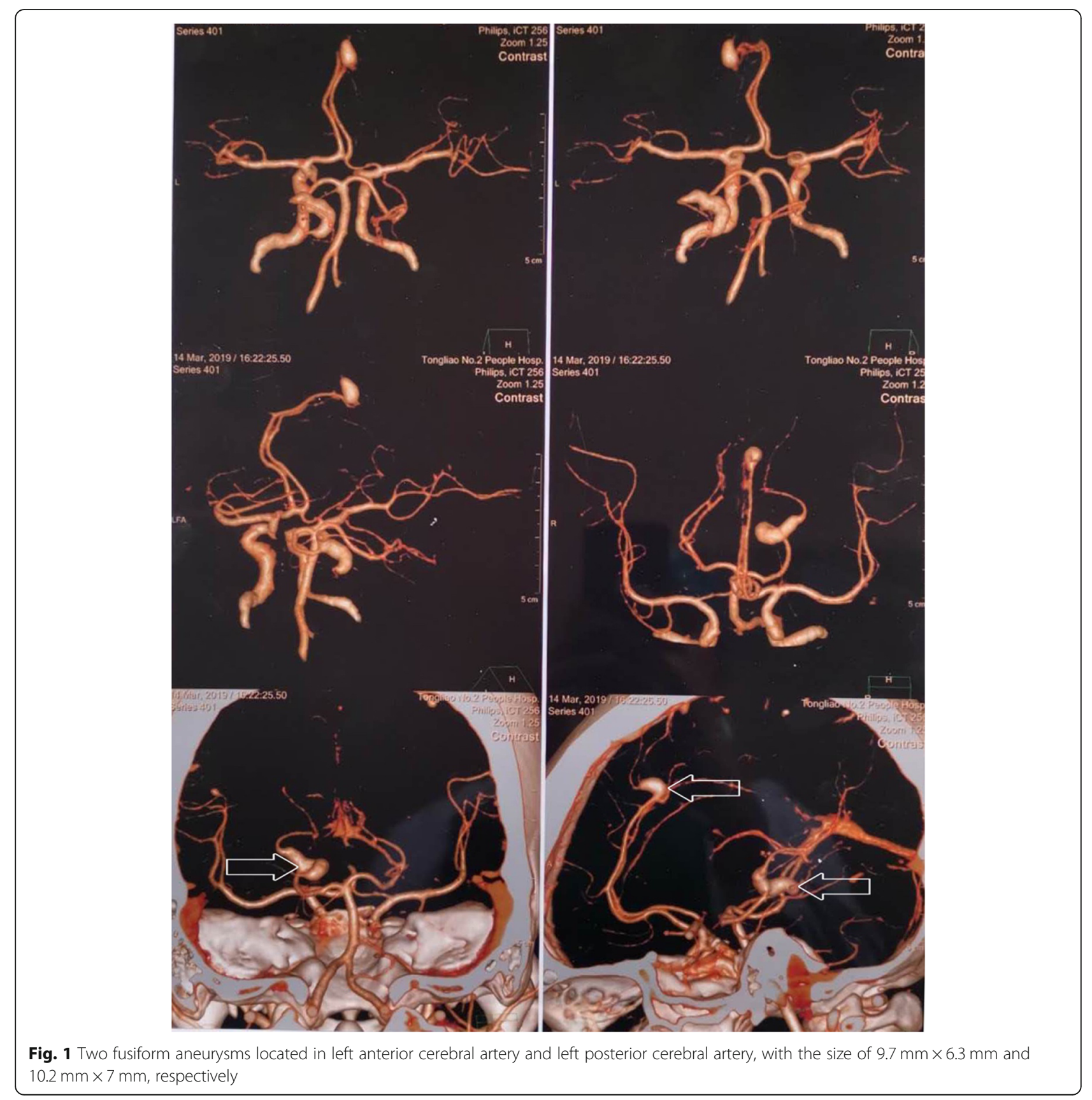




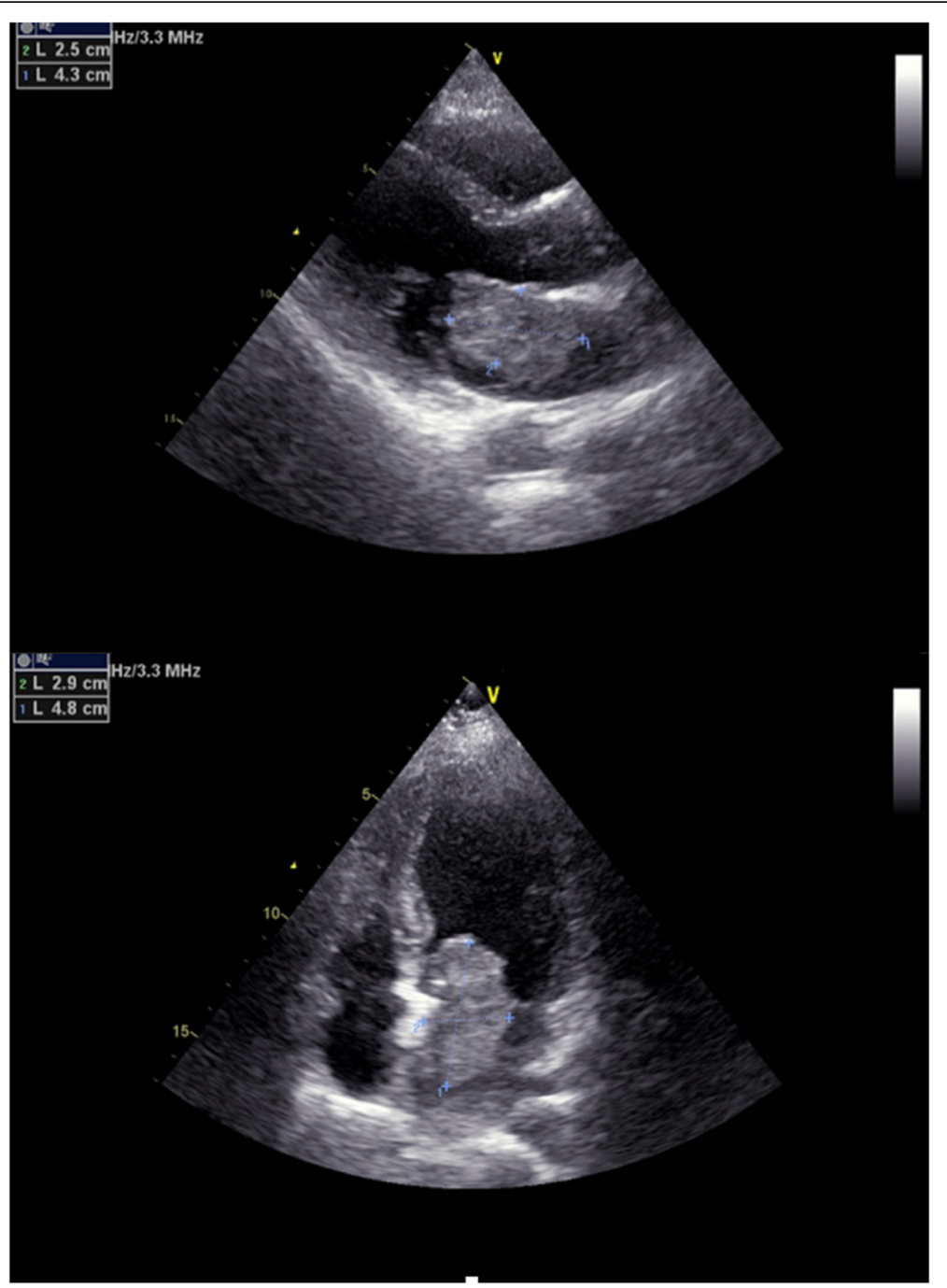

Fig. 2 TTE revealed a $4.8 \times 2.9 \times 2.5 \mathrm{~cm}^{3}$ mass attached to the interatrial septum in the left atrium, which obstructed the mitral orifice without mitral valve regurgitation

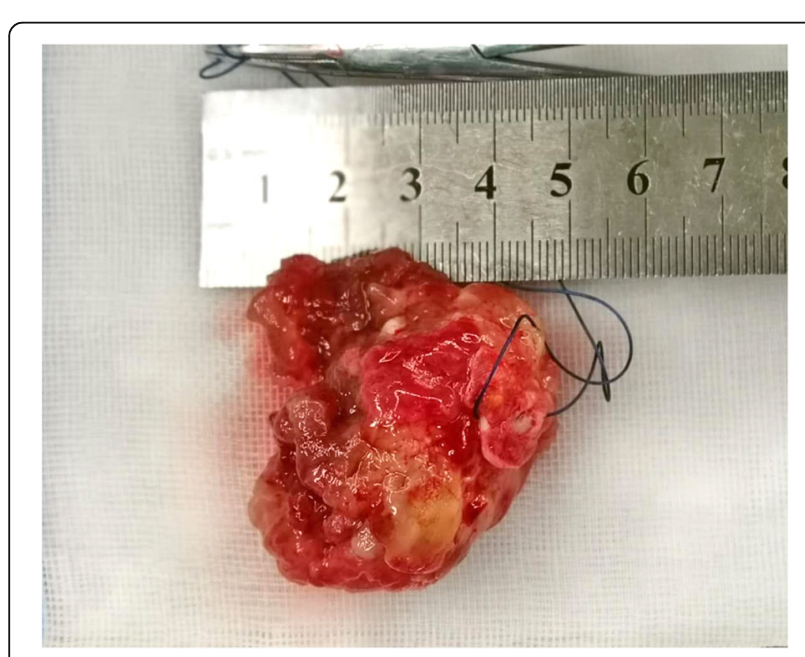

Fig. 3 Polypoid type of atrial myxoma continuous infusion of fentanyl $\left(0.3 \mu \mathrm{g} / \mathrm{kg} \times \mathrm{h}^{-1}\right)$ was performed to ensure analgesia and attenuate cardiovascular response to tracheal intubation. She was extubated $3 \mathrm{~h}$ after surgery without neurological disorder and discharged from ICU on the first day. Intravenous patient-controlled analgesia pump was employed to insure postoperative numeric rating scale (NRS) score lower than $3(0=$ No pain, $10=$ worst pain imaginable) [4]. She was fully recovered and discharged home on the sixth day after surgery.

\section{Review and analysis of the literature}

The keywords "cerebral aneurysm", "intracranial aneurysm", "myxoma", and "anesthesia" were used for searching in the PubMed and Google Scholar databases. The literature written in English published from January 1966 to April 2019 was reviewed, and articles or abstracts providing the following information were included, for instance, age, gender, 


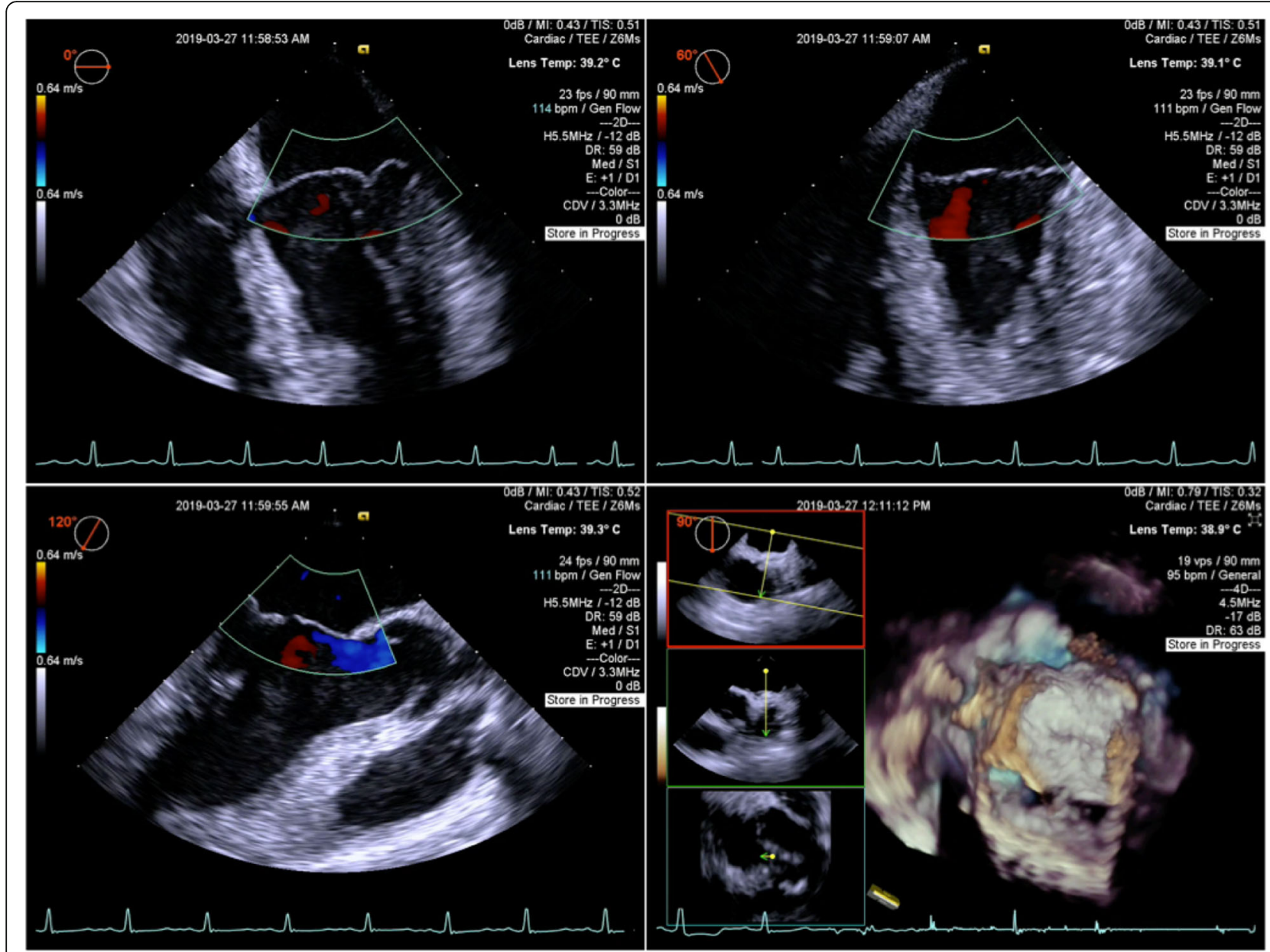

Fig. 4 After resection of myxoma, no mitral regurgitation or shunt flow across the atrial septum was revealed by transesophageal echocardiography

intervention for myxoma and aneurysm, complication, and outcome. Eventually, there were 47 reports of 49 cases and a total of 50 cases analyzed [3,5-49]. The median age was 38 years $(95 \% \mathrm{CI}, 34-42)$, and female/male ratio was 3.17:1. Resection of atrial myxoma was performed first in $90 \%$ (45) cases. Among these, conservative therapy for cerebral aneurysm was performed in 70\% (35) cases, including repeated operations of recurrence myxoma in 2 cases $[33,40]$. Whereas, craniotomy for aneurysm in 3 cases $[8,11,19]$, coiling for 2 cases [15, 34], radiation for 1 case [32], and cytostatic treatment for 1 case was carried out later [12]. Only one case reported craniotomy was performed first and early resection of myxoma was advised [13]. Three patients were dead in the early 1970s due to lack of knowledge and treatment $[45,46,48]$. After resection of myxoma, 13.3\% (6/45) patients suffered neurological dysfunction, while acute left hemiparesis appeared during induction of anesthesia and the operation was delayed in one case [5]. Severe neurological complication appeared in one patient with chronic renal failure, who finally died of sepsis [22]. No perioperative subarachnoid hemorrhage (SAH) was reported. Except in one patient, a conservative therapy was attempted, and a myxoma was verified by autopsy with cerebral aneurysms in 1973 [45]. During follow-up period, the rates of stable and regression of aneurysm were 50\% (25 cases) and 10\% (5 cases) respectively, while enlargement was $10 \%$ (5 cases), and new formation was $12 \%$ ( 6 cases). The subgroup of 11 progressive cases was further analyzed, continuous conservative therapy was performed in 4 cases, operation was carried out in 3 cases, and radiotherapy was administered in one case. Further follow-up revealed stable or regression after the treatment. Only one patient suffered SAH [21]. Although anesthesia management was introduced in only one case, it was in craniotomy procedure [13].

\section{Discussion and conclusions}

The incidence of primary heart tumors is less than $0.2 \%$ in patients. $75 \%$ of the tumors are benign, in which approximately $50 \%$ are myxomas [1]. Nearly three quarters of myxomas are located in the left atrium, while $15 \sim$ 
$20 \%$ are in the right atrium. Up to $20 \%$ of patients can be asymptomatic, whereas in a large case series, mitral valve obstruction, systemic emboli, and constitutional symptoms occurred [50, 51]. Systemic emboli has been well documented, especially embolic stroke $[52,53]$. It was reported a villous myxoma might be associated with more chances of metastasis of myxomas, and polypoid type was the only independent predictor of systemic emboli [54]. However, cerebral aneurysms related to atrial myxoma are rare. This patient was asymptomatic with myxoma, and neurological symptoms appeared first, for instance, dizziness and headache. The myxoma was polypoid type in this case.

In 1894, Marchand first reported an interesting phenomenon that cerebral aneurysms were associated with atrial myxoma [55]. Until 2005, Sabolek demonstrated the typical manifestation of aneurysms were multiple with fusiform shape [27]. To date, only around 50 case reports written in English could be found in the literature (Table 1). However, the exact mechanism is still not clear. The hypothesis of "Metastasize and Infiltrate" was considered as an essential mechanism for cerebral aneurysm formation. Myxoma cells may metastasize to the cerebral arteries, infiltrate into the vessel wall through the vasa vasorum or endothelial, interrupt the elastic lamina, and lead to aneurysm formation. Histological examination of the excised cerebral aneurysm verified this hypothesis $[29,36,48]$. Recent reports proposed another hypothesis, which is inflammation reaction arised from myxoma. It is reported that new cerebral aneurysms can form after myxoma resection, without recurrent myxoma or embolism [56]. Some studies found that new aneurysm formed with elevated proinflammation cytokines like interleukin-6 (IL-6) after resection of myxoma [27]. What is more, IL-6 level upregulated by myxoma may contribute to aneurysm formation [57, 58]. Other researches illuminated that IL- 6 could promote matrix metalloproteinases expression and activity, which enhance invasion of myxoma cells [23, 59]. Unfortunately, IL-6 level was not tested in our patient.

There are no clinical practice guidelines on such patients. Myxoma was suggested to be resected first to prevent systemic emboli and mitral valve obstruction $[1,10]$. In the meantime, fusiform aneurysm is not suitable for clipping or coiling compared to saccular aneurysm, surgical procedure is still an important intervention [60]. Fortunately, the SAH rate of multiple cerebral fusiform aneurysms related to atrial myxoma was low [27]. In addition, it is reported that the cerebral aneurysms regressed after myxoma resection in some cases $[3,5]$. Therefore, a conservative treatment approach for cerebral aneurysms was recommended by the preoperative MDT meeting.

Anesthesia management was an enormous challenge. Few piece of evidence was found in the database to guide optimal clinical anesthesia practice. The procedural strategy was to prevent ischemic and hemorrhagic stroke. Intraoperative cerebrovascular monitoring techniques remain controversial [61]. $\mathrm{PbtO}_{2}$ monitoring was recommended to detect brain ischemia and intracranial hypertension in neurocritical care patients [62]. As is known to all, the transmural pressure (TMP) of cerebral aneurysm is equal to cerebral perfusion pressure (CPP), which depends on mean arterial pressure (MAP) and intracranial pressure (ICP).

$$
\mathrm{TMP}=\mathrm{CPP}=\mathrm{MAP}-\mathrm{ICP}
$$

Therefore, an increase in MAP or a decrease in ICP will lead to an increase in CPP, which might increase the risk of rupture of aneurysm. On the contrary, a decrease in MAP or an increase in ICP will increase the risk of cerebral ischemia [63]. Firstly, induction of general anesthesia was an important step. One patient was reported to develop an acute left hemiparesis during induction [5]. Thus, it is crucial to control the TMP diligently. MAP and heart rate (HR) was recommended to close to baseline [64]. Lidocaine is beneficial to such patients, which could not only blunt cerebral hemodynamic response to endotracheal intubation, but also attenuate proinflammatory effects $[65,66]$. Besides, esmolol and fentanyl were demonstrated to prevent hemodynamic fluctuation related to intubation in a randomized controlled trial [67]. Secondly, cardiopulmonary bypass (CPB) is a risk factor of stroke, whose pathophysiological mechanisms refer to hemorrhagic, global ischemia, and embolic [68]. TEE plays a vital role in evaluating embolism originated from the heart [69]. On the other hand, it is instrumental to detect the pathogenesis of hypotension, guide fluid replenishment and identify mitral regurgitation and shunt flow [70]. With respect to $\mathrm{SAH}$, perioperative hypertension and anticoagulation are common in the cardiac surgery [68], which may increase the risk of aneurysm rupture. Although a most recent large observational study investigated the risk of postoperative 30-day SAH was $0.29 \%$, not higher than general population [71], it was suggested to decrease $\mathrm{CPB}$ time and intensively control the blood pressure [68]. In addition, $\mathrm{PaCO}_{2}$ should be maintained at normal level, and hyperventilation, which will decrease ICP, should be avoided [72]. In this case, the CPB time was $41 \mathrm{~min}$, fluctuation range of MAP was within $10 \%$, and $\mathrm{PaCO}_{2}$ was normal throughout the procedure. Thirdly, the fast track cardiac anesthesia was implemented to evaluate neurological function early after procedure, which aims to extubation within $1 \sim 6 \mathrm{~h}$ postoperation [73]. However, tracheal extubation should be paid more attention, when tachycardia, hypertension and coughing frequently occur [74]. And it would increase the risk of aneurysm rupture. Fentanyl attenuates cough and cardiovascular response effectively, which can be 
Table 1 Case reports of multiple aneurysms related to atrial myxoma

\begin{tabular}{|c|c|c|c|c|c|c|c|}
\hline & Author & Year & Age & Gender & Procedure (myxoma, aneurysm) & Complication & Outcome \\
\hline 1 & This case & 2019 & 38 & $\mathrm{~F}$ & Resection, Conservative & None & $\begin{array}{l}\text { Discharge } 6 \text { days postoperation and } \\
\text { follow-up }\end{array}$ \\
\hline 2 & $\begin{array}{l}\text { Coutinho R, } \\
\text { et al }\end{array}$ & 2018 & 46 & $\mathrm{~F}$ & Resection, Conservative & $\begin{array}{l}\text { Acute left hemiparesis } \\
\text { during induction }\end{array}$ & $\begin{array}{l}\text { Aneurysms completely regressed } \\
18 \text { days later and follow-up }\end{array}$ \\
\hline 3 & $\begin{array}{l}\text { Penn DL, } \\
\text { et al }\end{array}$ & 2018 & 12 & M & Resection, Conservative & None & $\begin{array}{l}1 \text { year follow-up, growth of } 2 \\
\text { aneurysms, hybrid procedure, } \\
43 \text { months follow-up, unchanged }\end{array}$ \\
\hline 4 & \multirow{2}{*}{$\begin{array}{l}\text { Flores PL, } \\
\text { et al }\end{array}$} & \multirow[t]{2}{*}{2018} & 61 & M & Resection, Conservative & None & 18 months follow-up, unchanged \\
\hline 5 & & & 19 & $\mathrm{~F}$ & Resection, Conservative & None & $\begin{array}{l}5 \text { year follow-up, several enlarged, } \\
\text { others regressed, asymptomatic, } \\
\text { conservative }\end{array}$ \\
\hline 6 & $\begin{array}{l}\text { Yoo HJ, } \\
\text { et al }\end{array}$ & 2018 & 20 & $\mathrm{~F}$ & Resection, Craniotomy later & Lost vision in right eye & Not mentioned \\
\hline 7 & $\begin{array}{l}\text { Quan K, } \\
\text { et al }\end{array}$ & 2017 & 49 & $\mathrm{~F}$ & Resection, Conservative & Not mentioned & $\begin{array}{l}\text { Further intracranial lesions resection } \\
\text { may be performed }\end{array}$ \\
\hline 8 & $\begin{array}{l}\text { Sveinsson } \mathrm{O} \text {, } \\
\text { et al }\end{array}$ & 2015 & 19 & $\mathrm{~F}$ & Resection, Conservative & None & 1 year follow-up, unchanged \\
\hline 9 & $\begin{array}{l}\text { Zheng J, } \\
\text { et al }\end{array}$ & 2015 & 25 & $\mathrm{~F}$ & $\begin{array}{l}\text { Resection first, craniotomy } 7 \\
\text { months later }\end{array}$ & $\begin{array}{l}\text { Drowsiness and partial } \\
\text { seizure } 6 \text { days after } \\
\text { craniotomy }\end{array}$ & $\begin{array}{l}\text { Discharge } 7 \text { days later, } 2 \text { months } \\
\text { follow-up, unchanged }\end{array}$ \\
\hline 10 & & 2015 & 39 & $\mathrm{~F}$ & Resection 20 years ago, Conservative & None & 14 months follow-up, occastional dizziness \\
\hline 11 & $\begin{array}{l}\text { Vontobel J, } \\
\text { et al }\end{array}$ & 2015 & 41 & $\mathrm{~F}$ & Resection, Cytostatic treatment & None & $\begin{array}{l}\text { Follow-up, decreased tracer uptake in PET, } \\
\text { stable aneurysm sizes }\end{array}$ \\
\hline 12 & $\begin{array}{l}\text { Srivastava S, } \\
\text { et al }\end{array}$ & 2014 & 30 & $\mathrm{~F}$ & None, Craniotomy first & None & $\begin{array}{l}\text { Discharge } 7 \text { days later, early resection } \\
\text { of myxoma was advised }\end{array}$ \\
\hline 13 & $X u Q$, et al & 2013 & 46 & $\mathrm{~F}$ & Resection, Conservative & None & Follow-up \\
\hline 14 & $\begin{array}{l}\text { Al-Said Y, } \\
\text { et al }\end{array}$ & 2013 & 67 & $\mathrm{~F}$ & Resection, Coiling 1 week later & None & 1 year follow-up, unchanged \\
\hline 15 & Kim $\mathrm{H}$, et al & 2012 & 58 & M & Resection, Conservative & None & 1 year follow-up, unchanged \\
\hline 16 & $\begin{array}{l}\text { KJ George, } \\
\text { et al }\end{array}$ & 2012 & 45 & $\mathrm{~F}$ & Resection, Conservative & None & $\begin{array}{l}\text { Discharge } 2 \text { weeks later, } 18 \text { months } \\
\text { follow-up, unchanged }\end{array}$ \\
\hline 17 & Lee $\mathrm{SJ}$, et al & 2012 & 55 & $\mathrm{~F}$ & Resection, Conservative & Not mentioned & 47 months follow-up, asymptomatic \\
\hline 18 & $\begin{array}{l}\text { Radoi MP, } \\
\text { et al }\end{array}$ & 2012 & 45 & $\mathrm{~F}$ & $\begin{array}{l}\text { Myxoma } 1 \text { year ago, Craniotomy } \\
\text { twice for } 2 \text { lesions }\end{array}$ & $\begin{array}{l}\text { Minor neurological } \\
\text { deficits }\end{array}$ & $\begin{array}{l}\text { Discharge } 3 \text { weeks later, } 12 \text { months } \\
\text { follow-up, unchanged }\end{array}$ \\
\hline 19 & $\begin{array}{l}\text { Chiang } \mathrm{KH} \text {, } \\
\text { et al }\end{array}$ & 2011 & 52 & $\mathrm{~F}$ & Resection, Conservative & None & 2 years follow-up, unchanged \\
\hline 20 & $\begin{array}{l}\text { Eddleman } \\
\text { CS, et al }\end{array}$ & 2010 & 18 & M & Resection, Conservative & Not mentioned & $\begin{array}{l}4 \text { months follow-up, multiple } \\
\text { aneurysms formated and hemmorrhage, } \\
3 \text { months later, several aneurysms enlarged } \\
\text { and hemmorrhage }\end{array}$ \\
\hline 21 & $\begin{array}{l}\text { Koo YH, } \\
\text { et al }\end{array}$ & 2009 & 65 & $\mathrm{~F}$ & Resection, Conservative & None & 6 months follow-up, unchanged \\
\hline 22 & $\begin{array}{l}\text { Shinn SH, } \\
\text { et al }\end{array}$ & 2009 & 48 & $\mathrm{~F}$ & Resection, Conservative & $\begin{array}{l}\text { Complex-focal type of } \\
\text { status epilepticus }\end{array}$ & Dead due to sepsis 22 days after surgery \\
\hline 23 & $\begin{array}{l}\text { Ryou KS, } \\
\text { et al }\end{array}$ & 2008 & 27 & $\mathrm{~F}$ & Resection, Conservative & Intermittent headache & 11 years follow-up, unchanged \\
\hline 24 & Li Q, et al & 2008 & 27 & $\mathrm{~F}$ & Resection, Conservative & None & 2 years follow-up, unchanged \\
\hline 25 & $\begin{array}{l}\text { Kvitting JP, } \\
\text { et al }\end{array}$ & 2008 & 55 & $\mathrm{~F}$ & Resection, Conservative & None & 6 months follow-up, unchanged \\
\hline 26 & $\begin{array}{l}\text { Sedat J, } \\
\text { et al }\end{array}$ & 2007 & 50 & $\mathrm{~F}$ & Resection, None & None & $\begin{array}{l}5 \text { years later aneurysms formated } \\
\text { and radiation therapy, } 1 \text { year follow-up, } \\
\text { one aneurysm regressed }\end{array}$ \\
\hline 27 & Namura $\mathrm{O}$, & 2007 & 35 & M & Resection, Conservative & Raynaud's phenomenon & 10 years follow-up, unchanged \\
\hline
\end{tabular}


Table 1 Case reports of multiple aneurysms related to atrial myxoma (Continued)

\begin{tabular}{|c|c|c|c|c|c|c|c|}
\hline & Author & Year & Age & Gender & Procedure (myxoma, aneurysm) & Complication & Outcome \\
\hline 28 & $\begin{array}{l}\text { Herbst M, } \\
\text { et al }\end{array}$ & 2005 & 31 & M & Resection, Conservative & None & 2 years follow-up, unchanged \\
\hline 29 & $\begin{array}{l}\text { Sabolek M, } \\
\text { et al }\end{array}$ & 2005 & 43 & $\mathrm{~F}$ & Resection, Conservative & None & $\begin{array}{l}15 \text { months follow-up, one aneurysm } \\
\text { regressed }\end{array}$ \\
\hline 30 & $\begin{array}{l}\text { Chen Z, } \\
\text { et al }\end{array}$ & 2005 & 19 & $\mathrm{~F}$ & Resection, None & None & $\begin{array}{l}2 \text { years later multiple aneurysms } \\
\text { formated and conservative therapy, } \\
1 \text { year follow-up, unchanged }\end{array}$ \\
\hline 31 & $\begin{array}{l}\text { Josephson } \\
\text { SA, et al }\end{array}$ & 2005 & 33 & $\mathrm{~F}$ & Not mentioned & Not mentioned & 8 years follow-up, unchanged \\
\hline 32 & $\begin{array}{l}\text { Ashalatha } R, \\
\text { et al }\end{array}$ & 2005 & 54 & M & Resection, None & None & $\begin{array}{l}6 \text { months follow-up, multiple } \\
\text { aneurysms formated and Conservative } \\
\text { therapy }\end{array}$ \\
\hline 33 & $\begin{array}{l}\text { Altundag MB, } \\
\text { et al }\end{array}$ & 2005 & 41 & $\mathrm{~F}$ & Resection, Radiation 1 year later & None & 4 years follow-up, unchanged \\
\hline 34 & $\begin{array}{l}\text { Stock K, } \\
\text { et al }\end{array}$ & 2004 & 22 & $\mathrm{~F}$ & Resection 2 times, conservative & None & $\begin{array}{l}11 \text { years follow-up, some aneurysms } \\
\text { regressed and some aneurysms smaller, } \\
\text { no new aneurysm }\end{array}$ \\
\hline 35 & $\begin{array}{l}\text { Yilmaz MB, } \\
\text { et al }\end{array}$ & 2003 & 38 & $\mathrm{~F}$ & $\begin{array}{l}\text { Recurrence and resection of } \\
\text { myxoma, coil embolization for one } \\
\text { giant aneurysm }\end{array}$ & None & Follow-up, unchanged \\
\hline 36 & $\begin{array}{l}\text { Furuya K, } \\
\text { et al }\end{array}$ & 1995 & 35 & M & Resection, Conservative & None & $\begin{array}{l}19 \text { months follow-up, enlarged } \\
\text { and craniotomy, another } 5 \text { months } \\
\text { follow-up, unchanged }\end{array}$ \\
\hline 37 & $\begin{array}{l}\text { Mattle HP, } \\
\text { et al }\end{array}$ & 1995 & 49 & M & Resection, Conservative & Not mentioned & $\begin{array}{l}5 \text { years follow up, aneurysm } \\
\text { formated } 3 \text { years after surgery, } \\
\text { demented } 5 \text { years later and } \\
\text { continuously progressed }\end{array}$ \\
\hline 38 & $\begin{array}{l}\text { Suzuki T, } \\
\text { et al }\end{array}$ & 1994 & 34 & M & Resection, Conservative & Not mentioned & $\begin{array}{l}\text { Follow-up, aneurysm formated } \\
2 \text { months after surgery, and enlarged } \\
5 \text { months later }\end{array}$ \\
\hline 39 & $\begin{array}{l}\text { Chen } \mathrm{HJ} \text {, } \\
\text { et al }\end{array}$ & 1993 & 68 & $\mathrm{~F}$ & Resection, Conservative & Not mentioned & $\begin{array}{l}\text { Craniotomy } 1 \text { year later, } 2 \text { years } \\
\text { follow-up, unchanged }\end{array}$ \\
\hline 40 & $\begin{array}{l}\text { Hung } P C \text {, } \\
\text { et al }\end{array}$ & 1992 & 10 & $\mathrm{~F}$ & Resection, Conservative & None & 8 months follow-up, unchanged \\
\hline 41 & $\begin{array}{l}\text { Bobo } H_{\text {, }} \\
\text { et al }\end{array}$ & 1987 & 15 & $\mathrm{~F}$ & $\begin{array}{l}\text { Resection four times for recurrent } \\
\text { myxoma, Conservative }\end{array}$ & None & 6 months follow-up, unchanged \\
\hline 42 & $\begin{array}{l}\text { Reed OM, } \\
\text { et al }\end{array}$ & 1986 & 25 & $\mathrm{~F}$ & Resection, Conservative & Not mentioned & $\begin{array}{l}12 \text { years follow-up, clip for a } \\
\text { large aneurysm } 9 \text { years later }\end{array}$ \\
\hline 43 & $\begin{array}{l}\text { Branch } C L \text {, } \\
\text { et al }\end{array}$ & 1985 & 53 & $\mathrm{~F}$ & Resection, Conservative & None & $\begin{array}{l}18 \text { months follow-up, one aneurysm } \\
\text { disappeared }\end{array}$ \\
\hline 44 & $\begin{array}{l}\text { Leonhardt } \\
\text { ET, et al }\end{array}$ & 1977 & 31 & M & Resection, Conservative & None & 2 months follow-up, unchanged \\
\hline 45 & $\begin{array}{l}\text { Damásio H, } \\
\text { et al }\end{array}$ & 1975 & 43 & $\mathrm{~F}$ & Resection, Conservative & None & 1 year follow-up, unchanged \\
\hline 46 & $\begin{array}{l}\text { Steinmetz EF, } \\
\text { et al }\end{array}$ & 1973 & 48 & $\mathrm{~F}$ & Conservative, Conservative & $\begin{array}{l}\text { SAH and hematoma } \\
\text { evacuation }\end{array}$ & $\begin{array}{l}\text { Dead } 2 \text { months later, autopsy } \\
\text { verified myxoma with cerebral aneurysms }\end{array}$ \\
\hline 47 & $\begin{array}{l}\text { Burton C, } \\
\text { et al }\end{array}$ & 1970 & 41 & $\mathrm{~F}$ & None, craniotomy first & Not mentioned & Dead in the first day after surgery \\
\hline 48 & New PF, et al & 1970 & 41 & $\mathrm{~F}$ & Resection, Conservative & None & 8 years follow-up, unchanged \\
\hline 49 & $\begin{array}{l}\text { Price } \mathrm{DL} \text {, } \\
\text { et al }\end{array}$ & 1970 & 21 & $\mathrm{~F}$ & Conservative, Conservative & Not mentioned & $\begin{array}{l}\text { Dead } 11 \text { months later, autopsy verified } \\
\text { myxoma with cerebral aneurysms }\end{array}$ \\
\hline 50 & $\begin{array}{l}\text { Stoane } L \text {, } \\
\text { et al }\end{array}$ & 1966 & 29 & M & Resection, Conservative & None & $\begin{array}{l}2 \text { months follow-up, slightly larger and } \\
\text { conservative therapy }\end{array}$ \\
\hline
\end{tabular}


safely used in fast track cardiac anesthesia [75-77]. Fortunately, refined perioperative anesthesia management was performed in this rare case, and the patient recovered uneventfully.

Atrial myxoma-related cerebral aneurysms are always multiple and in a fusiform shape in most occasions. Early resection of myxoma and conservative therapy of aneurysm is an optimal treatment. It is a great challenge to anesthesiologists to prevent stroke perioperatively. TEE and $\mathrm{PbtO}_{2}$ monitoring play an essential role in anesthesia management. Fast track cardiac anesthesia is safe and effective to early evaluate neurological function. Long term follow-up for "myxomatous aneurysms" is recommended. And outcome of most patients is excellent. Further study is needed to reveal the mechanism of atrial myxoma resulting in multiple cerebral aneurysms.

\section{Abbreviations \\ AMR: Atrial myxoma resection; MCAs: Multiple cerebral aneurysms; CTA: Computed tomography angiography; TTE: Transthoracic echocardiography; MDT: Multidisciplinary team; HR: Heart rate; MAP: Mean arterial pressure; TEE: Transesophageal echocardiography; PbtO2: Parenchymal brain oxygen; ICU: Intensive care unit; NRS: Numeric rating scale; SAH: Subarachnoid hemorrhage; IL-6: Interleukin-6; TMP: Transmural pressure; CPP: Cerebral perfusion pressure; ICP: Intracranial pressure; CPB: Cardiopulmonary bypass}

\section{Acknowledgements}

We would like to thank Dr. Gang Liu and Dr. ZQ Han from department of cardiac surgery for their understanding, supporting and collaborating in this case. We also thank DL Wang from department of neurosurgery for evaluating the patient and making some pertinent suggestions.

\section{Authors' contributions}

RZ implemented perioperative anesthesia management of the patient, analyze the literature and complete the draft manuscript. ZYT helped postoperative follow-up and the collection of clinical data. QQ drew up the anesthesia plan. FM analyzed the data of perioperative transesophageal echocardiography. YF revised the manuscript. All authors read and approved the final manuscript.

\section{Funding}

None.

\section{Availability of data and materials}

The datasets used and analysed during the current study are available from the corresponding author on reasonable request.

\section{Ethics approval and consent to participate}

Not applicable.

\section{Consent for publication}

The patient has given written consent for her personal or clinical details along with any identifying images to be published in this study.

\section{Competing interests}

The authors declare that they have no competing interests.

\section{Author details}

'Department of Anesthesiology, Peking University People's Hospital, No. 11 Xi Zhi Men Nan Da Jie, Xicheng District, Beijing, China. ${ }^{2}$ Department of Anesthesiology, Beth Israel Deaconess Medical Center, Boston, USA.
Received: 23 September 2019 Accepted: 25 May 2020

Published online: 04 July 2020

\section{References}

1. Reynen K. Cardiac myxomas. N Engl J Med. 1995;333(24):1610-7.

2. Lee VH, Connolly HM, Brown RD Jr. Central nervous system manifestations of cardiac myxoma. Arch Neurol. 2007;64(8):1115-20.

3. Sedat J, Chau Y, Dunac A, Gomez N, Suissa L, Mahagne MH. Multiple cerebral aneurysms caused by cardiac myxoma. A case report and present state of knowledge. Interv Neuroradiol. 2007;13(2):179-84.

4. Breivik H, Borchgrevink PC, Allen SM, Rosseland LA, Romundstad L, Hals EK, Kvarstein G, Stubhaug A. Assessment of pain. Br J Anaesth. 2008;101(1):17-24.

5. Renan Coutinho, MAyara Graciani, Livia Afonso, Thiago Teixiera, Beatriz Haynes, Carolina Oliviera, MArco Lima. Brain Aneurysm Associated with Atrial Myxoma Neurology April 10, 2018;90(15 Supplement):6.208.

6. Penn DL, Lanpher AB, Klein JM, Kozakewich HPW, Kahle KT, Smith ER, Orbach DB. Multimodal treatment approach in a patient with multiple intracranial myxomatous aneurysms. J Neurosurg Pediatr. 2018;21(3):315-21.

7. Flores PL, Haglund F, Bhogal P, Yeo Leong Litt L, Sodermann M. The dynamic natural history of cerebral aneurysms from cardiac myxomas: a review of the natural history of myxomatous aneurysms. Interv Neuroradiol. 2018;24(3):277-83.

8. Yoo HJ, Chung J, Ahn JS. Cerebral myxomatous aneurysm treated by M2M2 bypass: a case report. The Nerve. 2018;4(2):120-2.

9. Quan K, Song J, Zhu W, Chen L, Pan Z, Li P, Mao Y. Repeated multiple intracranial hemorrhages induced by cardiac myxoma mimicking cavernous angiomas: a case report. Chin Neurosurg J. 2017;3(1):3.

10. Sveinsson O, Herrman L. Multiple cerebral aneurysms in a patient with cardiac myxoma: what to do? BMJ Case Rep. 2015;2015.

11. Zheng J, Li S, Cao Y, Wang S, Wang R, Zhao J. Multiple cerebral myxomatous aneurysms: what is the optimal treatment? J Stroke Cerebrovasc Dis. 2015;24(1):232-8.

12. Vontobel J, Huellner M, Stolzmann P. Cerebral 'metastasizing' cardiac myxoma. Eur Heart J. 2016;37(21):1680.

13. Srivastava S, Tewari P. Stroke associated with left atrial mass: association of cerebral aneurysm with left atrial myxoma! Ann Card Anaesth. 2014;17(1):56-8.

14. Xu Q, Zhang X, Wu P, Wang M, Zhou Y, Feng Y. Multiple intracranial aneurysms followed left atrial myxoma: case report and literature review. J Thorac Dis. 2013;5(6):E227-31.

15. Al-Said Y, Al-Rached H, Baeesa S, Kurdi K, Zabani I, Hassan A. Emergency excision of cardiac myxoma and endovascular coiling of intracranial aneurysm after cerebral infarction. Case Rep Neurol Med. 2013;2013:839270.

16. Kim H, Park EA, Lee W, Chung JW, Park JH. Multiple cerebral and coronary aneurysms in a patient with left atrial myxoma. Int J Cardiovasc Imaging. 2012;28(Suppl 2):129-32.

17. George KJ, Rennie A, Saxena A. Multiple cerebral aneurysms secondary to cardiac myxoma. Br J Neurosurg. 2012;26(3):409-11.

18. Lee SJ, Kim JH, Na CY, Oh SS. Eleven years' experience with Korean cardiac myxoma patients: focus on embolic complications. Cerebrovasc Dis. 2012; 33(5):471-9.

19. Radoi MP, Stefanescu F, Arsene D. Brain metastases and multiple cerebral aneurysms from cardiac myxoma: case report and review of the literature. Br J Neurosurg. 2012;26(6):893-5.

20. Chiang K-H, Cheng H-M, Chang B-S, Chiu C-H, Yen P-S. Multiple cerebral aneurysms as manifestations of cardiac myxoma: brain imaging, digital subtraction angiography, and echocardiography. Tzu Chi Med J. 2011;23(2):63-5.

21. Eddleman CS, Gottardi-Littell NR, Bendok BR, Batjer HH, Bernstein RA. Rupture of cerebral myxomatous aneurysm months after resection of the primary cardiac tumor. Neurocrit Care. 2010;13(2):252-5.

22. Shinn SH, Chon SH, Kim HJ. Multiple cerebral aneurysms associated with cardiac myxoma in a patient with chronic renal failure: how can we resolve multiple cerebral aneurysms? Thorac Cardiovasc Surg. 2009;57(1):47-8.

23. Koo YH, Kim TG, Kim OJ, Oh SH. Multiple fusiform cerebral aneurysms and highly elevated serum interleukin-6 in cardiac myxoma. J Korean Neurosurg Soc. 2009;45(6):394-6.

24. Li Q, Shang H, Zhou D, Liu R, He L, Zheng H. Repeated embolism and multiple aneurysms: central nervous system manifestations of cardiac myxoma. Eur J Neurol. 2008;15(12):e112-3.

25. Kvitting JP, Engvall J, Broqvist M, Franzen S, Andersson M, Ohlsson U, Nielsen NE. Recurrence of myxoma in the left ventricle with concurrent cerebral fusiform aneurysms after previous atrial myxoma surgery. J Thorac Cardiovasc Surg. 2008;135(5):1172-3. 
26. Namura O, Saitoh M, Moro H, Watanabe H, Sogawa M, Nishikura K, Hayashi J. A case of biatrial multiple myxomas with glandular structure. Ann Thorac Cardiovasc Surg. 2007;13(6):423-7.

27. Sabolek M, Bachus-Banaschak K, Bachus R, Arnold G, Storch A. Multiple cerebral aneurysms as delayed complication of left cardiac myxoma: a case report and review. Acta Neurol Scand. 2005;111(6):345-50.

28. Josephson SA, Johnston SC. Multiple stable fusiform intracranial aneurysms following atrial myxoma. Neurology. 2005;64(3):526.

29. Herbst M, Wattjes MP, Urbach H, Inhetvin-Hutter C, Becker D, Klockgether T, Hartmann A. Cerebral embolism from left atrial myxoma leading to cerebral and retinal aneurysms: a case report. AJNR Am J Neuroradiol. 2005;26(3): 666-9.

30. Chen Z, Wang YL, Ye W, Miao ZR, Song QB, Ling F. Multiple intracranial aneurysms as delayed complication of atrial myxoma. Case report and literature review. Interv Neuroradiol. 2005;11(3):251-4.

31. Ashalatha R, Moosa A, Gupta AK, Krishna Manohar SR, Sandhyamani S. Cerebral aneurysms in atrial myxoma: a delayed, rare manifestation. Neurol India. 2005;53(2):216-8.

32. Altundag MB, Ertas G, Ucer AR, Durmus S, Abanuz H, Calikoglu T, Ozbagi K, Demirkasimoglu A, Kaya B, Bakkal BH, et al. Brain metastasis of cardiac myxoma: case report and review of the literature. J Neuro-Oncol. 2005;75(2):181-4.

33. Stock K. Multiple cerebral aneurysms in a patient with recurrent cardiac myxomas. A case report. Interv Neuroradiol. 2004;10(4):335-40.

34. Yilmaz MB, Akin Y, Guray U, Kisacik HL, Korkmaz S. Late recurrence of left atrial myxoma with multiple intracranial aneurysms. Int J Cardiol. 2003;87(23):303-5.

35. Mattle HP, Maurer D, Sturzenegger M, Ozdoba C, Baumgartner RW, Schroth G. Cardiac myxomas: a long term study. J Neurol. 1995;242(10):689-94.

36. Furuya K, Sasaki T, Yoshimoto Y, Okada Y, Fujimaki T, Kirino T, et al. J Neurosurg. 1995;83(1):170-3.

37. Suzuki T, Nagai R, Yamazaki T, Shiojima I, Maemura K, Yamaoki K, Tanaka N, Machida T, Kotsuka Y, Furuse A, et al. Rapid growth of intracranial aneurysms secondary to cardiac myxoma. Neurology. 1994;44(3 Pt 1):570-1.

38. Chen $\mathrm{HJ}$, Liou CW, Chen L. Metastatic atrial myxoma presenting as intracranial aneurysms with hemorrhage: case report. Surg Neurol. 1993;40(1):61-4.

39. Hung PC, Wang HS, Chou ML, Huang SC, Su WJ. Multiple cerebral aneurysms in a child with cardiac myxoma. J Formos Med Assoc. 1992;91(8): 818-21.

40. Bobo H, Evans OB. Intracranial aneurysms in a child with recurrent atrial myxoma. Pediatr Neurol. 1987;3(4):230-2.

41. Reed OM, Mellette JR Jr, Fitzpatrick JE. Cutaneous lentiginosis with atrial myxomas. J Am Acad Dermatol. 1986;15(2 Pt 2):398-402.

42. Branch CL Jr, Laster DW, Kelly DL Jr. Left atrial myxoma with cerebral emboli. Neurosurgery. 1985;16(5):675-80.

43. Leonhardt ET, Kullenberg KP. Bilateral atrial myxomas with multiple arterial aneurysms--a syndrome mimicking polyarteritis nodosa. Am J Med. 1977; 62(5):792-4.

44. Damasio H, Seabra-Gomes R, da Silva JP, Damasio AR, Antunes JL. Multiple cerebral aneurysms and cardiac myxoma. Arch Neurol. 1975;32(4):269-70.

45. Steinmetz EF, Calanchini PR, Aguilar MJ. Left atrial myxoma as a neurological problem: a case report and review. Stroke. 1973;4(3):451-8.

46. Price DL, Harris JL, New PF, Cantu RC. Cardiac myxoma. A clinicopathologic and angiographic study. Arch Neurol. 1970;23(6):558-67.

47. New PF, Price DL, Carter B. Cerebral angiography in cardiac myxoma. Correlation of angiographic and histopathological findings. Radiology. 1970;96(2):335-45.

48. Burton C, Johnston J. Multiple cerebral aneurysms and cardiac myxoma. N Engl J Med. 1970;282(1):35-6.

49. Stoane L, Allen JH Jr, Collins HA. Radiologic observations in cerebral embolization from left heart myxomas. Radiology. 1966;87(2):262-6.

50. Burke A, Jeudy J Jr, Virmani R. Cardiac tumours: an update: cardiac tumours. Heart. 2008;94(1):117-23.

51. Pinede L, Duhaut $P$, Loire R. Clinical presentation of left atrial cardiac myxoma. A series of 112 consecutive cases. Medicine (Baltimore). 2001;80(3):159-72.

52. Elbardissi AW, Dearani JA, Daly RC, Mullany CJ, Orszulak TA, Puga FJ, Schaff HV. Survival after resection of primary cardiac tumors: a 48-year experience. Circulation. 2008;118(14 Suppl):S7-15.

53. Aggarwal SK, Barik R, Sarma TC, lyer VR, Sai V, Mishra J, Voleti CD. Clinical presentation and investigation findings in cardiac myxomas: new insights from the developing world. Am Heart J. 2007;154(6):1102-7.

54. Ha JW, Kang WC, Chung N, Chang BC, Rim SJ, Kwon JW, Jang Y, Shim WH Cho SY, Kim SS, et al. Echocardiographic and morphologic characteristics of left atrial myxoma and their relation to systemic embolism. Am J Cardiol. 1999;83(11):1579-82 A1578.

55. Marchand F. Zur Kenntnis der Embolie und Thrombose der Gerhirnarterien, Zugleich ein Beitrag zur Casuistik der primaren Herztumoren und der gekreuzten Embolie. Klin Wochenschr. 1894;31:1-5.

56. Santillan A, Sigounas D, Fink ME, Gobin YP. Multiple fusiform intracranial aneurysms 14 years after atrial myxoma resection. Arch Neurol. 2012;69(9): 1204-5.

57. Jean WC, Walski-Easton SM, Nussbaum ES. Multiple intracranial aneurysms as delayed complications of an atrial myxoma: case report. Neurosurgery. 2001:49(1):200-2 discussion 202-203.

58. Ezerioha N, Feng W. Intracardiac Myxoma, cerebral aneurysms and elevated Interleukin-6. Case Rep Neurol. 2015;7(2):152-5.

59. Orlandi A, Ciucci A, Ferlosio A, Pellegrino A, Chiariello L, Spagnoli LG. Increased expression and activity of matrix metalloproteinases characterize embolic cardiac myxomas. Am J Pathol. 2005;166(6):1619-28.

60. Barletta EA, Ricci RL, Silva RDG, Gaspar R, Araujo JFM, Neves MWF, de Aquino JLB, Barba Belsuzarri TA. Fusiform aneurysms: a review from its pathogenesis to treatment options. Surg Neurol Int. 2018;9:189.

61. Gruber A, Dorfer C, Standhardt H, Bavinzski G, Knosp E. Prospective comparison of intraoperative vascular monitoring technologies during cerebral aneurysm surgery. Neurosurgery. 2011;68(3):657-73 discussion 673.

62. Oddo M, Bosel J, Participants in the International Multidisciplinary Consensus Conference on Multimodality M. Monitoring of brain and systemic oxygenation in neurocritical care patients. Neurocrit Care. 2014; 21(Suppl 2):S103-20.

63. Cottrell JE, SD. Anesthesia and neurosurgery. 4th ed. St. Louis: Mosby; 2001.

64. Abd-Elsayed AA, Wehby AS, Farag E. Anesthetic management of patients with intracranial aneurysms. Ochsner J. 2014;14(3):418-25.

65. Hamill JF, Bedford RF, Weaver DC, Colohan AR. Lidocaine before endotracheal intubation: intravenous or laryngotracheal? Anesthesiology. 1981;55(5):578-81.

66. Dunn LK, Durieux ME. Perioperative use of intravenous Lidocaine. Anesthesiology. 2017;126(4):729-37.

67. Ugur B, Ogurlu M, Gezer E, Nuri Aydin O, Gursoy F. Effects of esmolol, lidocaine and fentanyl on haemodynamic responses to endotracheal intubation: a comparative study. Clin Drug Investig. 2007;27(4):269-77.

68. Devgun JK, Gul S, Mohananey D, Jones BM, Hussain MS, Jobanputra Y, Kumar A, Svensson LG, Tuzcu EM, Kapadia SR. Cerebrovascular events after cardiovascular procedures: risk factors, recognition, and prevention strategies. J Am Coll Cardiol. 2018;71(17):1910-20.

69. Celeste F, Muratori M, Mapelli M, Pepi M. The evolving role and use of echocardiography in the evaluation of cardiac source of embolism. J Cardiovasc Echogr. 2017;27(2):33-44.

70. Reeves ST, Finley AC, Skubas NJ, Swaminathan M, Whitley WS, Glas KE, Hahn RT, Shanewise JS, Adams MS, Shernan SK, et al. Basic perioperative transesophageal echocardiography examination: a consensus statement of the American Society of Echocardiography and the Society of Cardiovascular Anesthesiologists. J Am Soc Echocardiogr. 2013;26(5):443-56.

71. Nam JS, Jeon SB, Jo JY, Joung KW, Chin JH, Lee EH, Chung $\mathrm{CH}$, Choi IC. Perioperative rupture risk of unruptured intracranial aneurysms in cardiovascular surgery. Brain. 2019;142(5):1408-15.

72. Yao F. Yao and Artusio's anesthesiology. 6th ed. Philadelphia: Lippincott 2008.

73. Cheng DC. Fast track cardiac surgery pathways: early extubation, process of care, and cost containment. Anesthesiology. 1998;88(6):1429-33.

74. Hartley M, Vaughan RS. Problems associated with tracheal extubation. Br J Anaesth. 1993;71(4):561-8.

75. Tagaito $Y$, Isono S, Nishino T. Upper airway reflexes during a combination of propofol and fentanyl anesthesia. Anesthesiology. 1998;88(6):1459-66.

76. Nishina K, Mikawa K, Maekawa N, Obara H. Fentanyl attenuates cardiovascular responses to tracheal extubation. Acta Anaesthesiol Scand. 1995;39(1):85-9.

77. Myles PS, Daly DJ, Djaiani G, Lee A, Cheng DC. A systematic review of the safety and effectiveness of fast-track cardiac anesthesia. Anesthesiology. 2003:99(4):982-7.

\section{Publisher's Note}

Springer Nature remains neutral with regard to jurisdictional claims in published maps and institutional affiliations. 